\title{
Yod
}

Revue des études hébraïques et juives

$17 \mid 2012$

La presse écrite au Moyen-Orient

\section{La presse jeune-turque en Égypte et la modernité (1895-1908)}

Young Turks' Press in Egypt and the Modernity (1895-1908)

העיתונות של הטורקים הצעירים במצרים והמודרניזם (1895 עד 1908)

\section{Wajda Sendesni}

\section{(2) OpenEdition}

\section{Journals}

Édition électronique

URL : https://journals.openedition.org/yod/1576

DOI : $10.4000 /$ yod. 1576

ISSN : 2261-0200

\section{Éditeur}

INALCO

Édition imprimée

Date de publication : 30 juin 2012

Pagination : 25-36

ISBN : 978-2-85831-200-9

ISSN : 0338-9316

\section{Référence électronique}

Wajda Sendesni, « La presse jeune-turque en Égypte et la modernité (1895-1908) », Yod [En ligne], 17| 2012, mis en ligne le 27 octobre 2012, consulté le 08 juillet 2021. URL : http:// journals.openedition.org/yod/1576 ; DOI : https://doi.org/10.4000/yod.1576

Ce document a été généré automatiquement le 8 juillet 2021.

\section{(c) $(1)$ ( $)$}

Yod est mis à disposition selon les termes de la Licence Creative Commons Attribution - Pas d'Utilisation Commerciale 4.0 International. 


\title{
La presse jeune-turque en Égypte et la modernité (1895-1908)
}

\author{
Young Turks' Press in Egypt and the Modernity (1895-1908) \\ העיתונות של הטורקים הצעירים במצרים והמודרניזם (1895 עד 1908)
}

\section{Wajda Sendesni}

1 Né officiellement en 1895 , le mouvement jeune-turc est un mouvement d'opposition au régime du sultan Abdülhamid II et favorable au rétablissement de la constitution ottomane suspendue par le sultan en 1878. Les Jeunes-Turcs sont diplômés pour la plupart de l'École de médecine (Tibbiye), de l'École d'administration (Mülkiye), de l'École militaire (Harbiye) d'Istanbul ou ont fait leurs études en Europe. Ils mènent leurs activités contre le régime hamidien depuis leur exil dans les capitales européennes et en Égypte en se servant essentiellement de la presse comme outil de propagande. À côté des questions politiques, le débat portait souvent dans leurs journaux sur la modernité et les moyens de l'introduire dans l'Empire. En Égypte, les Jeunes-Turcs et les libéraux arabes ne conçoivent pas tous de la même manière cette modernité, mais ils pensent unanimement que l'avenir de l'Empire ottoman dépend de l'éducation et du progrès des sciences. Ils estiment que le savoir est la meilleure arme dont peuvent se servir les Ottomans pour rattraper le retard vis-à-vis de l'Europe.

\section{Le savoir, clé du progrès}

2 Pour les Jeunes-Turcs, tous les maux de l'Empire découlent directement ou indirectement de l'ignorance que seule l'éducation, ce remède magique, peut guérir. Elle permet de barrer la route à l'impérialisme, de s'imprégner de patriotisme, de s'opposer au despotisme.

3 Même les ulémas prennent conscience de l'importance de l'éducation et de la nécessité d'étudier les sciences et les arts modernes. Dans son livre Umm al-Qûra, le cheikh 'Abd al-Rahman al-Kawakibi, un réformiste musulman syrien qui a fini ses jours en Égypte, dit à propos de la situation dans le monde musulman au XIX ${ }^{e}$ siècle : «La cause du 
désordre actuel réside dans l'ignorance générale ${ }^{1}$. Il déplore l'abandon de l'étude des sciences par les musulmans et y voit la cause principale de leur décadence. Tombés dans l'ignorance, les musulmans deviennent des ennemis de la science et ils taxent d'hérésie quiconque veut s'y consacrer ${ }^{2}$. Le secret du progrès de l'Occident est percé, dit Kawakibi: c'est $\mathrm{du}$ fait de cultiver les sciences au point d'en faire «un soleil indispensable à tout être vivant $»^{3}$ qu'il a pu aller si loin sur le chemin de la civilisation, et les musulmans doivent suivre son exemple. En tant qu'uléma, Kawakibi n'hésite pas à accorder plus d'importance aux sciences « profanes » parce qu'il les juge plus utiles que les sciences religieuses.

4 Son attitude rejoint celle du Jeune-Turc Abdullah Cevdet, mais ce dernier va plus loin que Kawakibi. Il ne se contente pas de critiquer les sciences religieuses, il doute également de l'utilité de l'observance des devoirs religieux. Il se moque des hodjas et des cheikhs qui croient, chaque fois qu'une calamité s'abat sur les pays musulmans, qu'il s'agit d'un châtiment divin dû à leurs péchés et il se demande : pourquoi ce châtiment n'est-il pas infligé aux mécréants qui sont encore plus impies que les musulmans? Pourquoi Dieu leur donne-t-il prospérité et victoire à eux? La réponse n'est pas à chercher, selon lui, dans les livres de théologie mais dans les sciences et les arts qui font la force de l'Occident.

En vérité, l'observance des devoirs religieux et une vie pieuse ont leurs mérites spéciaux, mais ces mérites ne sont pas efficaces pour préserver les possessions d'une nation ni pour contribuer aux victoires [...]. Combien de centaines d'arts et de sciences ne sont-elles pas employées de nos jours dans l'administration des pays et dans les services militaires et maritimes. Assurément, personne ne peut s'attendre à ce qu'une religion enseigne toutes ces centaines d'arts ou de sciences; personne ne peut raisonnablement admettre que le Coran et les traditions promettent la victoire qui est remportée à l'aide de ces centaines d'arts et de sciences, par la simple observance de la religion et la conduite dans le chemin du devoir ${ }^{4}$.

\section{Pas de science sans morale?}

5 Necmeddin Arif, Jeune-Turc d'Égypte et médecin ayant fait ses études à Paris, s'installe à partir de 1901 au Caire où il ouvre un cabinet et exerce son métier. En 1904, il décide de publier un guide pratique de l'éducation à Paris qui fournit des informations sur le système éducatif français afin qu'il soit utile à ses compatriotes qui voudraient aller faire leurs études dans cette ville ainsi qu'aux réformateurs ottomans.

En tant que médecin, Necmeddin Arif ne peut rester insensible au progrès de la science en Occident et plus particulièrement à Paris qu'il dit être « un soleil de science et de savoir $»^{5}$. Pourtant, cet homme imprégné de savoir moderne, conscient du pouvoir magique de la science et ayant fréquenté des écoles occidentales où la raison règne en maitresse absolue, plaide pour la morale indispensable au bon fonctionnement de la société. Il compare la science à un bateau dont la morale serait le gouvernail. Sans gouvernail, le bateau irait à la dérive.

La science, le savoir et l'art ne suffisent pas. On peut même dire qu'ils ne sont pas du tout suffisants. S'ils ne sont pas imprégnés, accompagnés de morale (ahlak), le bonheur des hommes est impossible. Si on compare la science et le savoir à un bateau, la morale en serait le gouvernail. Tout comme un bateau sans gouvernail se trouve exposé aux dangers et ne peut atteindre sa destination, les sciences et les arts qui ne s'accompagnent pas de morale ne peuvent arriver à bon port ${ }^{6}$.

7 Tout comme il admire Paris qui rayonne par son savoir, Necmeddin Arif critique la décadence morale qui y règne et il met en garde ses compatriotes qui, en allant dans 
cette ville pour s'instruire, perdraient leurs bonnes mœurs orientales. Il leur conseille alors de ne prendre de l'Europe que son savoir tout en rejetant le reste :

Allons en Europe, mais pas pour être gagnés, c'est-à-dire perdre nos bonnes mours, allons-y pour gagner. Nos rapports avec les Européens doivent être comme notre rapport avec le feu. Profitons de leur lumière, de leur chaleur mais ne pénétrons pas à l'intérieur d'eux [sinon] nous brûlerons?

8 Tout en étant conscients de la nécessité des sciences et des arts de l'Occident pour faire progresser l'Empire, les réformistes ottomans restent très moralisateurs, ils ont peur que la modernité efface leur identité. Ils insistent donc sur les bonnes mœurs, le code moral oriental, la vertu..., autant de freins pour prévenir les « dérapages » de la science.

Le Syrien Rafiq al-'Azm, Jeune-Turc et futur arabiste qui a reçu une formation d'uléma et grandi dans un milieu conservateur, n'est pas moins conscient de la nécessité de la science et de ses bienfaits que Necmeddin Arif, mais il se méfie encore plus que lui d'une science sans brides.

Chaque fois que la science progresse chez un peuple, il lui devient plus facile d'élever les âmes et de les polir, surtout si la science est doublée de vertu; et sa vertu est d'être mise en pratique par le savant qui sait forcément le bien et le mal qui résulte de chaque acte 8 .

Pour être vertueuse, la science doit être maniée par un savant consciencieux qui s'en sert uniquement pour le bien, pour servir ses intérêts et ceux des gens de sa race. C'est que la science se trouve au cœur de la lutte entre civilisations que Rafiq al-'Azm considère comme une lutte pour la survie dans le sens darwinien du terme :

L'homme ne peut réussir ce qu'il entreprend que grâce à la science exacte qui est l'évolution de l'esprit au point de discerner les causes du bonheur et du malheur et de comprendre [le principe] de la lutte pour la vie qui consiste en la survie du plus fort aux dépens du plus faible. L'esprit ne peut atteindre ce degré de savoir que par l'apprentissage et l'éducation, à condition que ce savoir soit conforme à la vertu de sorte que l'apprenant sente qu'il apprend ce qui peut lui être utile à lui et aux gens de sa race [...] Et sachez que le savoir est la balance qui permet l'égalité des forces entre les peuples en conflit dans le domaine de la civilisation. Lorsque l'un des antagonistes s'arrête de travailler [pour le progrès] alors que l'autre continue, le second devance forcément le premier et triomphe de lui dans la lutte pour la survie 9 .

11 Cette guerre ou lutte entre civilisations est ce qui caractérise les rapports entre l'Orient et l'Occident à cette époque d'impérialisme européen et d'expansion coloniale. Le progrès des sciences et le renforcement de l'Empire constituent la seule garantie contre l'impérialisme européen.

12 Mais tout comme elles servent à se protéger de l'ennemi extérieur, les sciences permettent de combattre la tyrannie. Rien ne fait plus peur au tyran que le savoir et les savants. Le tyran sait que si le voile de l'ignorance est déchiré, cela sonnera le glas de son règne. C'est ce qu'explique Kawakibi dans son livre sur la nature du despotisme ${ }^{10}$. Les souverains ignorants et tyranniques, voilà l'obstacle essentiel à l'union des musulmans. Pour le surmonter, Rafiq al-'Azm appelle les musulmans à deux choses : d'abord cultiver les sciences, s'instruire, s'éclairer, multiplier les écoles, aller faire des études en Europe... Et une fois armés de savoir, ils doivent faire la guerre au despotisme et aux despotes et réclamer liberté et régime constitutionnel ${ }^{11}$.

13 Un autre Jeune-Turc d'Égypte, Frah Antoun, croit que l'éducation qui est capable de guérir les maux de l'Empire peut aussi fournir le ciment qui souderait les différents éléments de la nation ottomane. C'est sur les bancs de l'école publique que s'apprend la fraternité. Farah Antoun, qui consacre une rubrique de sa revue Al-Djami'a à 
l'éducation, partage la responsabilité de l'éducation de la jeunesse ottomane entre la mère, l'école et la presse. Mais en fin de compte, le rôle revient principalement à l'école, car les mères capables d'élever des enfants attachés à leur patrie doivent être elles-mêmes bien éduquées. De même, les journalistes qui dirigeraient une presse de qualité capable d'élever la conscience nationale au-dessus de tous les clivages ne peuvent le faire que s'ils ont reçu une bonne éducation au préalable.

Les moyens de fraternité en Orient sont au nombre de trois : la mère à la maison, le maître à l'école et les journaux dans la rue. S'il se trouvait dans les foyers des mères pour semer dans les esprits des enfants de l'Orient, dès leur jeune âge, les bases du vrai patriotisme et des nobles principes, s'il existait des écoles nationales où l'éducation serait de qualité et obligatoire pour accueillir les différents éléments de la nation qui s'assoiraient sur le même banc, recevraient la même éducation et le même traitement et puis s'il se trouvait des journaux libres ou sous contrôle qui guideraient cette nouvelle génération avec honnêteté et impartialité [...], si ces choses honorables se trouvaient réunies, la fraternité régnerait, la haine disparaîtrait et il pousserait dans le champ oriental, ottoman plus exactement, de nouvelles plantes pour lesquelles le soleil de la liberté politique serait lumière et non pas $\mathrm{feu}^{12}$.

\section{L'islam est-il incompatible avec la science?} l'adapter à la modernité. Pour eux, ce n'est pas l'islam dans son essence qui est incompatible avec le progrès, mais la fausse interprétation de ses textes et de ses commandements. Il n'est donc pas question de renoncer à la religion pour gagner en modernité, mais de l'épurer, de la réformer, de la réinterpréter et de l'adapter à son époque.

18 Les Jeunes-Turcs n'ont pas les mêmes rapports à l'islam que les réformistes musulmans. Ils sont beaucoup plus influencés, voire fascinés, par la philosophie matérialiste. La plupart d'entre eux ont séjourné à Paris à un moment ou un autre de leur exil, ils ont 
fréquenté les cercles intellectuels parisiens, ont lu les œuvres des positivistes, des darwinistes. Ce que les autres appellent modernité, eux le confondent avec la science.

Pour les Jeunes-Turcs, la science est la religion de l'élite et la religion est la science des masses ${ }^{14}$. Derrière la lourde rhétorique islamique qui caractérise leur discours, et qui fait croire à une similarité entre leur pensée et celle des réformistes musulmans, se cache un projet de modernisation basé sur les principes de la philosophie matérialiste. Le discours islamique est une façade qui sert à éviter de choquer le public musulman auquel s'adressent les Jeunes-Turcs et à endormir la méfiance de leurs alliés parmi les ulémas. Ils s'emploient à habiller les emprunts à la civilisation occidentale en idées et usages trouvant leur origine dans les principes de l'islam, comme le résument ces propos d'Abdullah Cevdet dans sa revue Ictihad:

Nous avons constaté par nos longues expériences que l'esprit musulman fermera toute ouverture à la clarté si elle vient immédiatement du monde chrétien. Il nous faut donc, à nous qui assumons le soin de transfuser un sang nouveau dans les veines musulmanes, de chercher et trouver tous les principes progressistes dans l'institution de l'islam même, et l'islamisme en déborde. Telle est la raison qui nous amène souvent à parler des musulmans et de l'islamisme ${ }^{15}$.

Le but de certains Jeunes-Turcs n'est pas de concilier modernité et islam, mais de remplacer le second par la première. En cherchant dans la religion musulmane ce qui permet de légitimer les innovations qu'ils veulent introduire dans l'Empire ottoman, ils essaient par tous les moyens de manipuler leurs lecteurs au point de leur faire croire que l'islam n'est rien d'autre qu'une forme de matérialisme ${ }^{16}$. Au fond, Ahmed Riza rêve de voir les musulmans se convertir au positivisme, tandis qu'Abdullah Cevdet, moins radical, considère que la conversion dans un premier temps des Ottomans au bahaïsme ${ }^{17}$ suffit, en attendant qu'ils soient assez mûrs pour adopter la science comme seule religion.

\section{Des médecins pour la société ?}

21 Abdullah Cevdet est, parmi les Jeunes-Turcs, le plus influencé par la philosophie matérialiste. Médecin, il s'intéresse à tout ce qui se rapporte à la médecine, à la psychologie, à la biologie, à la physiologie... Il adopte aussi bien le darwinisme que les théories des races et se passionne, entre autres, pour les écrits de Gustave Le Bon dont il dit vouloir traduire en turc l'ensemble des œuvres qu'il croit être d'une grande utilité.

À partir du second numéro de la revue Ictihad, A. Cevdet commence la publication en feuilleton du livre de G.Le Bon Lois psychologiques de l'évolution des peuples. Dans l'introduction à cette traduction, il évoque ses études à la faculté de médecine et ses débuts comme médecin lorsqu'il a réalisé la différence entre la théorie et la pratique. Il dit avoir compris alors qu'il ne suffit pas de savoir diagnostiquer les maladies pour les guérir et que la médecine n'a pas réponse à tout. Cela le plonge dans le désespoir et lui donne un sentiment d'impuissance. Il se dit que s'il avait étudié auparavant les lois profondes de l'évolution des maladies, il n'en serait pas arrivé là, car les maladies ont un rapport avec l'évolution de chaque peuple, avec l'état de la société. C'est pour cela qu'il y a besoin de médecins de la société capables de disséquer l'anatomie des peuples et d'étudier leur physiologie. A. Cevdet dit avoir pensé longtemps à cette question et ne 
sachant comment apporter une aide à son peuple et à l'humanité sur ce plan, il a cru utile de traduire le livre de Le Bon qui en traite en profondeur.

À mon avis, il est absurde et enfantin de vouloir s'attaquer au domaine difficile des réformes politiques et sociales sans connaitre les textes et les lois sociales contenus dans ce livre, tout comme il est absurde et enfantin de se prétendre médecin sans connaître la chirurgie et la physiologie ${ }^{18}$.

Les Jeunes-Turcs veulent des médecins pour la société, des hommes capables de diagnostiquer ses maux et d'y appliquer le bon traitement. La médecine, tout comme elle permet d'analyser le social, permet de comprendre le politique. Dans le journal Doğru Söz, publié au Caire à partir de 1906, un certain Dr Vedid parle de la composition biologique des corps vivants, en particulier celui des mammifères dont l'homme. En décortiquant le mode de fonctionnement et la distribution des rôles à l'intérieur de ce système, l'auteur en arrive à le comparer à un peuple, à un corps social très organisé où chacun doit jouer son rôle sans empiéter sur le domaine des autres car si cela se produit ou si l'un des organes dysfonctionne, tout le système est mis en péril. L'auteur conclut que même le corps humain semble être régi par un mode constitutionnel, ce qui invite à réfléchir et à tirer des leçons ${ }^{19}$.

Alors que les Jeunes-Turcs médecins parlent de la société en empruntant le lexique de l'anatomie et de la physiologie, un homme de lettres, le journaliste et poète Ahmed Kemal, soumet la langue à la théorie de l'évolution de Darwin. Dans un article du journal Doğru Söz intitulé "Mulahazât-i mütevaliye, lisan hakkında », il explique que conformément à la loi de l'évolution, la langue ne peut que changer. La forme, le style et l'orthographe évoluent à travers le temps, mais pas de manière fantaisiste. Chaque écrivain ou poète n'est pas libre d'innover dans la langue ou de la modifier selon sa volonté. L'évolution obéit à des règles, tout comme l'évolution des êtres vivants est régie par une loi qui prend en compte le passé et le présent ${ }^{20}$. Ahmed Kemal semble plaider pour une réforme de la langue ottomane, mais sans rupture avec le passé et le présent. Cela signifie peut-être qu'il ne veut pas d'un abandon de l'alphabet arabe mais d'une simplification de l'orthographe et d'une modification de la forme, c'est-à-dire un allègement du style.

Ahmed Kemal évoque dans cet article la décadence morale de l'Occident et cite au passage Max Nordau et son livre Dégénérescence ${ }^{21}$. Le fait que Nordau était également médecin rend sa position encore plus intéressante pour les Jeunes-Turcs car son discours est fondé sur la science. En évoquant son livre, Ahmed Kemal sous-entend qu'il partage l'avis de l'auteur concernant la dégénérescence morale de l'art et de la société dans l'Europe moderne.

Ahmed Kemal, qui semble avoir été influencé par le livre de Max Nordau, reprend et traduit dans son journal Doğru Söz l'enquête parue en France dans la Revue des revues autour de la question « Peut-il y avoir une morale sans Dieu? ", question à laquelle ont répondu des philosophes, des sociologues, des écrivains, des journalistes célèbres. Il s'agit de savoir si l'homme et la société peuvent se passer de religion et si une morale découlant de la science et se basant sur la raison peut remplacer la morale religieuse ${ }^{22}$. Le journal jeune-turc a cru utile de traduire cette « enquête » et d'y ajouter les avis de savants orientaux. Malheureusement, n'ayant pas la collection complète du journal, je n'ai pu voir que les deux premiers extraits de cette traduction. Je me demande donc si la traduction a été achevée et s'il y a eu la réponse des savants de l'Orient à la question ! Et comment ont-ils répondu à Stuart Mill, Lüdwig Büchner, Auguste Comte, Ferdinand 
Brunetière, Max Nordau, Charles Gide... ? Et Ahmed Kemal le traducteur : à quelle thèse adhère-t-il ? Probablement à celle qui dit que la morale et la religion sont indissociables en opposition à la thèse des philosophes matérialistes qui croient que la société doit se débarrasser de la morale religieuse pour en créer une autre fondée sur la raison.

Les Jeunes-Turcs veulent tous moderniser l'Empire ottoman pour qu'il ressemble à l'Europe « civilisée » et échappe à son sort de malade condamné à mort. Mais ils sont en même temps conscients de l'identité de cet empire oriental et musulman. Ils savent qu'ils ne peuvent pas parler ouvertement de matérialisme et d'abandon de la religion alors qu'ils s'adressent à une société composée à $90 \%$ de musulmans. D'ailleurs, les Jeunes-Turcs eux-mêmes sont majoritairement musulmans.

Au-delà du souci de soigner les apparences pour continuer à apparaître comme de "bons musulmans" aux yeux de leurs coreligionnaires, les Jeunes-Turcs croient sincèrement au potentiel de l'islam en tant que facteur de cohésion sociale. L'islam permet une certaine union et constitue un élément identitaire très fort. Cette identité islamique, les Jeunes-Turcs l'utilisent au gré des circonstances. Ils la brandissent contre l'impérialisme occidental, en jouent dans leur lutte contre le régime d'Abdülhamid et s'en servent dans la construction du nationalisme turc.

\section{NOTES}

1. Norbert Tapiéro, Les idées réformistes d'al-Kawakibi, Paris, Les éditions arabes, 1956.

2. Ibid., p. 60.

3. Ibid.

4. Abdullah Cevdet, « Musulmans debout », Ictihad supplément français, avril 1906.

5. Necmeddin Arif, Paris'te tahsil, Le Caire, 1904, p. 5.

6. Ibid., p. 8.

7. Ibid., p. 13.

8. Rafiq al-'Azm, Kitab al-Dûrûs al-Hikmiyya, leçon 23 Al-tarbiya wa al-akhlâq ("L'éducation et la morale »), Le Caire, 1898, p. 75.

9. Ibid., leçon 22 Al-'ilm bil-'amal (« L'utilisation du savoir »), p. 72-73.

10. Kawakibi, Tabai‘ al-istibdâd, Le Caire, Matba'at al-Dustur al-'Uthmanî, [s.d.], p. 33-34: «Le tyran n'ignore pas qu'il ne peut y avoir servitude et despotisme que si le peuple est stupide, plongé dans les ténèbres de l'ignorance et l'égarement de l'aveuglement. Si le tyran était un oiseau, il aurait été une chauve-souris chassant parmi la masse dans l'obscurité de l'ignorance et s'il était un animal sauvage, il aurait été un loup dévorant les volailles des villes sous le voile de la nuit. Le savoir est une étincelle de la lumière divine. Dieu a créé la lumière comme source d'éclairage, génératrice de chaleur et de force; de même il a fait du savoir un guide qui montre le bien et dénonce le mal, faisant naître une chaleur dans les âmes et une sagacité dans les esprits [...] Le tyran, tout comme il déteste le savoir pour ses conséquences, le déteste aussi en soi car il a un pouvoir supérieur à tout autre pouvoir. Le tyran se sent donc inférieur chaque fois qu'il se retrouve en face de quelqu'un plus savant que lui. »

11. Rafiq al-'Azm, Al-Djami'a al-Islamiyya wa Ûruppa, Le Caire, 1907, p. 77.

12. «Al-Ikhâ’ wa al-hurriyya », Al-Djami'a Al-'Uthmaniyya nº 3, 15 avril 1899, p. 2. 
13. Ernest Renan, L'islam et la science, avec la réponse d'Afghani, Éditions L'Archange Minotaure, 2003, p. 39-40 : «En vérité, la religion musulmane a cherché à étouffer la science et à en arrêter les progrès. Elle a réussi ainsi à enrayer le mouvement intellectuel ou philosophique et à détourner les esprits de la recherche de la vérité scientifique. Pareille tentative, si je ne me trompe, a été faite par la religion chrétienne et les chefs vénérés de l'Église catholique n'ont point encore désarmé que je sache. Ils continuent à lutter énergiquement contre ce qu'ils appellent l'esprit de vertige et d'erreur. Je sais toutes les difficultés que les musulmans auront à surmonter le même degré de civilisation, l'accès de la vérité à l'aide des procédés philosophiques et scientifiques leur étant interdit. "

14. M. Ş. Hanioğlu, TheYoung Turks in Opposition, Oxford University Press, 1995, p. 201.

15. Abdullah Cevdet, « Une profession de foi », Ictihad n 6, mai 1905.

16. M. ş. Hanioğlu, op.cit., p. 202.

17. Bahaïsme : secte dérivée du chiisme qui a vu le jour en Iran durant la seconde moitié du XIXE SIÈCLE, ELLE EST BASÉE SUR LE PRINCIPE D'UNION DE L'HUMANITÉ ET DÉFEND À SES ADEPTES L'ADOPTION D'UNE FOI AVEUGLE. IL FAUT QU'IL Y AIT UN LIEN ENTRE LA FOI ET LA RAISON, UNE HARMONIE ENTRE LA SCIENCE ET LA RELIGION.

18. Abdullah Cevdet, « Ruh-ul akvâm », Ictihad n 2 , janvier 1905, p. 2.

19. Dr Vedid, «Bir az da fen ve siyaset », Doğru Söz $n^{\circ} 12,13$ septembre 1906, p. 4.

20. Doğru Söz n ${ }^{\circ}$ 2, 13 septembre 1906.

21. Max Nordau (Budapest 1849-Paris 1923) médecin juif hongrois, cofondateur avec Herzl de l'Organisation sioniste mondiale, est aussi un critique sociologique qui s'est intéressé à ce qu'il appelle «la société de fin de siècle » en Europe et aux phénomènes qui étaient en train de la changer en profondeur. Dans son livre le plus connu, Dégénérescence, il prend une position moralisatrice contre l'art dégénéré qui affecte directement la société et décrit, en termes médicaux, ce phénomène qui traduit selon lui la volonté du monde « moderne » d'échapper aux règles morales qui le gouvernent. Nordau considère l'antisémitisme comme un résultat de cette dégénérescence morale.

22. « Ahlak Allahsız olur mu ? », Doğru Söz n 5, 17 mai 1906 et nº 10, 26 juillet 1906.

\section{RÉSUMÉS}

L'Égypte devient à la fin du XIX ${ }^{\mathrm{e}}$ siècle un foyer important du développement de la presse. Des Syriens et des Turcs fuyant la répression du régime hamidien s'y réfugient. Ils publient au Caire des journaux en arabe, en turc ou bilingues dans lesquels ils critiquent le sultan Abdülhamid et son régime et réclament le rétablissement de la constitution ottomane suspendue en 1878. Mais dans ces journaux, on peut aussi déceler un intérêt pour la modernité et le progrès qui apparaît à travers le nombre important d'articles qui y sont consacrés ainsi qu'à la science, à l'éducation, à la littérature, etc. Pour la jeune élite ottomane, plus que du changement de régime, l'avenir de l'Empire dépend de sa capacité à se transformer et à se moderniser car seule la modernité peut le sauver de la chute en permettant une réforme économique et sociale en profondeur.

Egypt in the late nineteenth century became an important center of development of the press. Syrians and Turks fleeing the Hamidian repressive regime take refuge there. They publish newspapers in Cairo in Arabic, Turkish or bilingual where they criticize the sultan Abdulhamid and his regime and demand the restoration of the Ottoman constitution suspended in 1877. But 
in these papers, one can also detect an interest in modernity and progress that appears throw articles on modernity, progress, science, education, literature, etc. For the young Ottoman elite, rather than regime change, the future of the empire depends on its ability to transform and modernize because only modernity can save it from collapse by providing a social and economic reform.

INDEX

Thèmes : littérature

Keywords : Press, Young Turks, Islam, modernity, science, progress, Egypt

\section{מילות מפתח}

ללחוץ, טורקים צעירים, סלאם, חדשנות, מדע, התקדמות, מצרים, ספרות:

Mots-clés : presse, Jeunes-Turcs, islam, modernité, science, progrès

Index géographique : Égypte 